\title{
ULTRASONIDO Y ANISOTROPÍA EN MADERA DE Thuja plicata Y Acer saccharum
}

\section{ULTRASOUND AND ANISOTROPY OF Thuja plicata AND Acer saccharum WOOD}

\author{
Javier Ramón Sotomayor-Castellanos ${ }^{1, \star}$, José María Villaseñor-Aguilar $^{I}$
}

\begin{abstract}
RESUMEN
La velocidad del ultrasonido en la madera y el módulo de elasticidad, siguen un patrón anisotrópico, que se infiere mediante un modelo teórico. El objetivo de la investigación fue determinar la variación de la velocidad del ultrasonido y del módulo de elasticidad en el plano longitudinal-tangencial en la madera de Thuja plicata y Acer saccharum. Se estudiaron 32 probetas de cada especie diseñadas específicamente para la investigación. Se calcularon la velocidad del ultrasonido y el módulo de elasticidad en diez posiciones en el plano longitudinal-tangencial. Los resultados experimentales se contrastaron con las previsiones del modelo de anisotropía propuesto. Para la madera de T. plicata y A. saccharum, las velocidades de ultrasonido fueron para la dirección longitudinal $5610 \mathrm{~m} / \mathrm{s}$ y 5935 $\mathrm{m} / \mathrm{s}$ y para la dirección tangencial $1198 \mathrm{~m} / \mathrm{s}$ y $1372 \mathrm{~m} / \mathrm{s}$. Los módulos de elasticidad en la dirección longitudinal fueron $11126 \mathrm{MPa}$ y $24688 \mathrm{MPa}$ y para la dirección tangencial $530 \mathrm{MPa}$ y $1320 \mathrm{MPa}$.
\end{abstract}

Palabras clave: Densidad, ensayos no destructivos, módulo de elasticidad, propiedades de la madera, velocidad de onda.

\begin{abstract}
The ultrasound speed and the modulus of elasticity of the wood material follow an anisotropic behavior predicted by a theoretical model. The goal of the research was to determine the variation of the ultrasound speed and the modulus of elasticity over the longitudinal-tangential plane of Thuja plicata and Acer saccharum wood. For each species, 32 wood specimens were tested. The ultrasound speed and the modulus of elasticity were calculated in ten positions over the longitudinal-tangential plane. Experimental results were contrasted with the predictions of the proposed anisotropy model. For the T. plicata and A. saccharum woods, the ultrasound speeds were, for the longitudinal direction $5610 \mathrm{~m} / \mathrm{s}$ and $5935 \mathrm{~m} / \mathrm{s}$, and for the tangential direction $1198 \mathrm{~m} / \mathrm{s}$ y $1372 \mathrm{~m} / \mathrm{s}$. The moduli of elasticity were, for the longitudinal direction $11126 \mathrm{MPa}$ and $24688 \mathrm{MPa}$, and for the tangential direction 530 $\mathrm{MPa}$ and $1320 \mathrm{MPa}$.
\end{abstract}

Keywords: Density, modulus of elasticity, non-destructive tests, wave speed, wood properties.

\footnotetext{
${ }^{1}$ Universidad Michoacana de San Nicolás de Hidalgo. Ciudad Universitaria, Morelia, Michoacán, México.

•Autor para correspondencia: madera999@yahoo.com 


\section{INTRODUCCIÓN}

El ultrasonido es una técnica de carácter no destructivo que se emplea para caracterizar mecánicamente a la madera. Si se combina la velocidad del ultrasonido con la densidad de la madera, se puede determinar su módulo de elasticidad (Keunecke et al. 2007). Independientemente de la variabilidad natural al interior y entre especies, la velocidad de onda es sensible al contenido de humedad de la madera (Ozyhar et al. 2013) y a su anisotropía material (Vázquez et al. 2015). Cuando disminuye el contenido de humedad de la madera, la velocidad del ultrasonido aumenta. Igualmente, si se modifica la orientación de la solicitación respecto a las direcciones de anisotropía de la madera, la velocidad del ultrasonido es diferente (De Oliveira et al. 2005).

Para la dirección longitudinal, se ha observado un rango que va de $4000 \mathrm{~m} / \mathrm{s}$ a $6000 \mathrm{~m} / \mathrm{s}$. Para la dirección radial, este intervalo es de $2000 \mathrm{~m} / \mathrm{s}$ a $2500 \mathrm{~m} / \mathrm{s}$. Finalmente, para la dirección tangencial, la velocidad de ultrasonido en la madera se encuentra en el rango de $1000 \mathrm{~m} / \mathrm{s}$ a $1800 \mathrm{~m} / \mathrm{s}$ (Bucur 2006). De tal forma, que cuando se mide la velocidad de onda en la madera, se observa un fenómeno de anisotropía. Caso interesante es la variación de la velocidad de onda entre las direcciones ortogonales. El enfoque teórico más común es el de predecir este cambio empleando un modelo que relaciona el parámetro correspondiente a la dirección longitudinal, con el respectivo a la dirección radial o tangencial. Esta ecuación combina las funciones trigonométricas seno y coseno elevados a un exponente, el cual es propio para cada especie y ha sido llamado modelo de anisotropía (Kabir 2001):

$$
C M_{\theta}=\frac{C M_{L} C M_{T}}{C M_{L} \operatorname{sen}^{n} \theta+C M_{T} \cos ^{n} \theta}
$$

Donde $C M_{\theta}$ es la característica mecánica para el ángulo $\theta, C M_{L}$ es la característica mecánica en la dirección longitudinal (L), $C M_{T}$ es la característica mecánica en la dirección tangencial (T), $\theta$ es el ángulo entre las direcciones longitudinal y tangencial y $\mathrm{n}$ es la constante experimental a determinar para cada especie y características mecánicas en cuestión.

La madera de $T$. picata y A. saccharum se emplea en componentes de edificación, muebles y artículos de alto valor agregado. Sus propiedades tecnológicas están descritas en el Manual de la Madera (Forest Products Laboratory 2010). Las especies de los géneros Thuja (gimnospermas) y Acer (angiospermas) se utilizan también en la elaboración de instrumentos musicales (Brémaud et al. 2012). Sus velocidades de ultrasonido están reportadas por Bucur (2006). Sin embargo, es difícil encontrar información sobre la anisotropía para las especies T. plicata y A. saccharum utilizando ultrasonido.

Para diseño de una pieza o componente estructural de un instrumento musical, es necesario conocer con precisión, entre otros parámetros, la densidad, la velocidad del ultrasonido y el módulo de elasticidad correspondiente (Wegst 2006). Actualmente, con el empleo generalizado de métodos de análisis numérico, se hace también necesaria información detallada de parámetros de ingeniería y su variación según las direcciones de anisotropía de la madera (Tankut et al. 2014).

Para incorporar especies de madera en la fabricación de objetos de alto valor agregado, es necesario determinar sus características tecnológicas considerando el carácter anisotrópico (Nadir et al. 2014). Esta caracterización encuentra también aplicación en el análisis numérico (Sonderegger et al. 2013) y para la concepción de productos asistida por computador (Mackerle 2005). Varias geometrías de probetas se han utilizado para determinar las características elásticas de la madera para las tres direcciones de anisotropía: radial, tangencial y longitudinal (Ozyhar et al. 2013, Gonçalvez et al. 2014). Los investigadores citados proponen protocolos experimentales que determinan las doce constantes elásticas correspondientes a las tres direcciones de ortotropía y a sus planos resultantes.

Existen escasos trabajos que realizan en probetas circulares (Figura 1) mediciones secuenciales en el intervalo de los ejes longitudinal y tangencial. Yanagida et al. (2007) efectúan una tomografía computarizada en probetas circulares de madera de Robinia psedoacacia. Con el objeto de validar 
un método para detectar defectos empleando ultrasonido, los autores presentan velocidades para varias direcciones, pero éstas no coinciden necesariamente con las direcciones de anisotropía de la madera. Empleando mapas bidimensionales de contorno de onda acústica Xu et al. (2014) estudian la anisotropía de la velocidad del ultrasonido en madera de Populus ussuriensis y emplean una función parabólica de segundo orden propuesta por Kabir (2001). De esta manera, comparan sus resultados con los previstos por la fórmula de Hankinson (Armstrong et al. 1991), modelo similar al propuesto en esta investigación.

La investigación plantea como hipótesis de trabajo que en la madera, la velocidad del ultrasonido y el módulo de elasticidad siguen un patrón anisotrópico, que puede ser inferido con un modelo teórico. Esta propuesta está limitada para el plano formado por las direcciones longitudinal y tangencial del plano leñoso, y es particular para el caso de estudio de una muestra estadísticamente representativa de madera de A. saccharum y T. plicata. Para verificar la hipótesis, el objetivo de la investigación fue determinar con ultrasonido la anisotropía de la madera de T. plicata y A. saccharum, calculando la velocidad del ultrasonido y el módulo de elasticidad en el plano longitudinal-tangencial.

\section{MATERIALES Y MÉTODOS}

Se recolectaron 20 piezas de madera de Thuja plicata L. y 20 de Acer saccharum Marshall, con dimensiones de 0,04 $\mathrm{m}$ de espesor por 0,20 $\mathrm{m}$ de ancho y 0,4 $\mathrm{m}$ de largo, alineadas de tal forma que las dimensiones correspondieron a las direcciones radial, tangencial y longitudinal del plano leñoso. $\mathrm{La}$ madera fue acondicionada durante 4 años en una cámara con una temperatura de $20^{\circ} \mathrm{C}\left( \pm 2{ }^{\circ} \mathrm{C}\right)$ y una humedad relativa del aire de $65 \%( \pm 2 \%)$, hasta que su peso fue constante. A partir de las piezas de madera fueron recortadas 35 probetas de forma circular (Figura 1). El diámetro de las probetas de $T$. plicata fue de $0,15 \mathrm{~m}$ y para $A$. saccharum fue de $0,125 \mathrm{~m}$. El espesor fue de $0,015 \mathrm{~m}$ para las probetas de las dos especies.

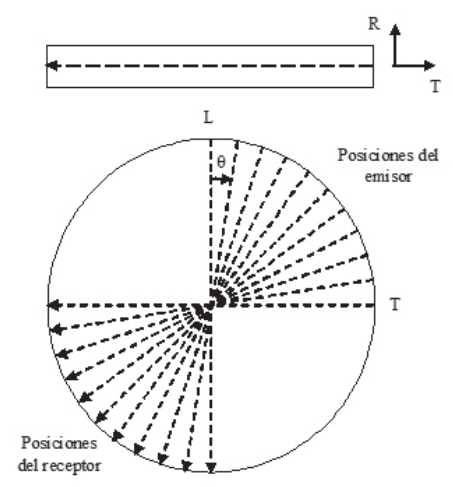

Figura 1. Diagrama de la probeta y direcciones de medición. $\Delta \theta=10^{\circ}$, ángulo de rotación de las mediciones, $\mathrm{L}=$ Dirección longitudinal $\left(\theta=0^{\circ}\right), \mathrm{T}=$ Dirección tangencial $\left(\theta=90^{\circ}\right), \mathrm{R}=$ Dirección radial. Las flechas discontinuas indican la dirección del ultrasonido.

La densidad de la madera $\left(\rho_{\mathrm{CH}}\right)$, correspondiente a un contenido de humedad $(\mathrm{CH})$, se calculó con la relación peso al momento del ensayo/volumen al momento del ensayo. La densidad básica $\left(\rho_{0}\right)$ se determinó en un grupo complementario de probetas, recortadas de las mismas piezas de madera y con dimensiones de $0,015 \mathrm{~m}$ por $0,015 \mathrm{~m}$ por $0,04 \mathrm{~m}$. El cálculo se efectuó con el cociente del peso de la madera en estado anhidro y el volumen de ésta en estado saturado, adaptando la norma ISO 130612:2014 (ISO 2014b). El contenido de humedad de las probetas al momento del ensayo se determinó con 
la relación peso al momento del ensayo en relación al peso de la probeta en estado seco, adaptando la norma ISO 13061-1:2014 (ISO 2014a).

Las pruebas de ultrasonido consistieron en medir la velocidad de transmisión del ultrasonido y determinar la velocidad del ultrasonido, entre dos posiciones en los extremos del plano longitudinaltangencial de las probetas (Figura 1), empleando el aparato sylvatest ${ }^{\circledR}$, con frecuencia de $22 \mathrm{KHz}$. En la primera posición se colocó el sensor emisor del ultrasonido y en la segunda, se colocó el sensor receptor. La primera medición se realizó en la dirección longitudinal de la probeta $\left(\theta=0^{\circ}\right)$. A continuación, se realizaron 10 mediciones con una rotación de 10 grados, en el sentido de las manecillas del reloj, cada una en el plano longitudinal-tangencial, de tal forma, que después de diez mediciones consecutivas, la última midió el tiempo de transmisión en la dirección tangencial $\left(\theta=90^{\circ}\right)$. La velocidad de onda se calculó con el cociente de la distancia entre los sensores emisor-receptor y el tiempo medido por el aparato. El módulo de elasticidad se calculó con la fórmula (2) propuesta por Pellerin y Ross (2002), donde: $E_{u s}=$ Módulo de elasticidad $(\mathrm{MPa}) ; \rho_{C H}=$ Densidad $\left(\mathrm{kg} / \mathrm{m}^{3}\right) ; \mathrm{y} v_{u s}=$ Velocidad del ultrasonido $(\mathrm{m} / \mathrm{s})$ :

$$
E_{u s}=\rho_{C H} v_{u s}^{2}
$$

Para contrastar los resultados del modelo de anisotropía, con los experimentales, se ajustaron los datos experimentales al modelo no lineal de anisotropía utilizando técnicas estadísticas de regresión, empleando un método no ponderado y siguiendo el algoritmo Scaled Levenberg-Marquardt con una tolerancia de 0,0001 también se determinaron los intervalos de confianza para las medias muestrales utilizando un nivel de confianza del $95 \%$. Para el ajuste de los modelos de regresión se siguieron las hipótesis de que para cada ángulo $\theta$ utilizado, las velocidades del ultrasonido y los módulos de elasticidad presentaron distribuciones normales y que las mediciones realizadas de la velocidad del ultrasonido y del módulo de elasticidad son independientes entre sí.

\section{RESULTADOS Y DISCUSIÓN}

La Tabla 1 presenta los resultados del contenido de humedad $(\mathrm{CH})$ y de la densidad al momento del ensayo $\left(\rho_{C H}\right)$. El contenido de humedad promedio de ambas especies indica que la madera estuvo seca al momento del ensayo. Los coeficientes de variación del contenido de humedad menores que 1,25 son considerados bajos para un material como la madera, lo que justifica que esta variable no influyera en los resultados. La madera de T. plicata es considera como de baja densidad, mientras que la madera de A. saccharum se considera como de densidad media, de acuerdo con Sotomayor y Ramírez (2013). Al igual que para el contenido de humedad, el coeficiente de variación de la densidad al momento del ensayo se considero bajo de acuerdo con Sotomayor y Ramírez (2014), lo que justifica que esta variable no fuera considerada en los resultados. 
Tabla 1. Contenido de humedad y densidad.

\begin{tabular}{|c|c|c|c|c|c|c|}
\hline & $\mathrm{CH}$ & $\boldsymbol{\rho}_{0}$ & $\boldsymbol{\rho}_{\mathrm{CH}}$ & $\mathrm{CH}$ & $\boldsymbol{\rho}_{0}$ & $\boldsymbol{\rho}_{\mathrm{CH}}$ \\
\hline & $(\%)$ & $\left(\mathrm{kg} / \mathrm{m}^{3}\right)$ & $\left(\mathrm{kg} / \mathrm{m}^{3}\right)$ & $(\%)$ & $\left(\mathrm{kg} / \mathrm{m}^{3}\right)$ & $\left(\mathrm{kg} / \mathrm{m}^{3}\right)$ \\
\hline & \multicolumn{3}{|c|}{ Thuja plicata } & \multicolumn{3}{|c|}{ Acer saccharum } \\
\hline $\bar{x}$ & 11,51 & 298 & 332 & 10,39 & 612 & 676 \\
\hline$\sigma$ & 0,14 & 15,18 & 20,30 & 0,12 & 80,28 & 91,85 \\
\hline $\mathrm{CV}$ & 1,23 & 5,10 & 6,12 & 1,15 & 13,12 & 13,59 \\
\hline
\end{tabular}

$\mathrm{CH}=$ Contenido de humedad; $\rho_{0}=$ Densidad básica; $\rho_{\mathrm{CH}}=$ Densidad a un contenido de humedad CH; $\bar{x}=$ Media; $\sigma=$ Desviación estándar; $\mathrm{CV}=$ Coeficiente de variación en porciento.

La Tabla 2 presenta las velocidades del ultrasonido y los módulos de elasticidad. Las magnitudes de los parámetros experimentales fueron similares a los reportados empleando técnicas de ultrasonido en la dirección longitudinal de la madera por Bucur (2006) para A. saccharum: $\rho_{12}=760 \mathrm{~kg} / \mathrm{m}^{3}, v_{u s}=$ $3800 \mathrm{~m} / \mathrm{s}$ y $E_{u s}=11000 \mathrm{MPa}$. Por su parte Brémaud et al. (2012) reportan para T. plicata: $\rho_{12}=390 \mathrm{~kg} /$ $\mathrm{m}^{3}, v_{u s}=4416 \mathrm{~m} / \mathrm{s}$ y $E_{u s}=7605 \mathrm{MPa}$, valores similares a los de esta investigación.

Tabla 2. Velocidad del ultrasonido y módulo de elasticidad.

\begin{tabular}{|c|c|c|c|c|c|c|c|c|}
\hline & \multicolumn{5}{|c|}{ Thuja plicata } & \multicolumn{4}{c|}{ Acer saccharum } \\
\hline$\theta$ & $\mathrm{v}_{\text {us }}$ & $\mathrm{v}_{\text {us }}$ & $\mathrm{E}_{\text {us }}$ & $\mathrm{E}_{\text {us }}$ & $\mathrm{v}_{\text {us }}$ & $\mathrm{v}_{\text {us }}$ & $\mathrm{E}_{\text {us }}$ & $\mathrm{E}_{\text {us }}$ \\
\hline$\left({ }^{\circ}\right)$ & $(\mathrm{m} / \mathrm{s})$ & $(\mathrm{m} / \mathrm{s})$ & $(\mathrm{MPa})$ & $(\mathrm{MPa})$ & $(\mathrm{m} / \mathrm{s})$ & $(\mathrm{m} / \mathrm{s})$ & $(\mathrm{MPa})$ & $(\mathrm{MPa})$ \\
\hline & Experimental & Teórico & Experimental & Teórico & Experimental & Teórico & Experimental & Teórico \\
\hline $0=\mathrm{L}$ & 5610 & 5610 & 11126 & 11133 & 5935 & 5936 & 24688 & 24693 \\
\hline 10 & 4966 & 5107 & 8648 & 8900 & 4860 & 4968 & 16559 & 17129 \\
\hline 20 & 4010 & 4009 & 5621 & 4691 & 3680 & 3716 & 9495 & 8688 \\
\hline 30 & 3150 & 2992 & 3468 & 2450 & 2769 & 2808 & 5374 & 4841 \\
\hline 40 & 2521 & 2270 & 2225 & 1460 & 2193 & 2218 & 3370 & 3101 \\
\hline 50 & 2098 & 1801 & 1537 & 989 & 1815 & 1843 & 2308 & 2229 \\
\hline 60 & 1842 & 1505 & 1188 & 745 & 1620 & 1607 & 1839 & 1757 \\
\hline 70 & 1642 & 1326 & 945 & 615 & 1469 & 1465 & 1513 & 1495 \\
\hline 80 & 1466 & 1229 & 763 & 550 & 1411 & 1392 & 1396 & 1362 \\
\hline $90=\mathrm{T}$ & 1198 & 1198 & 530 & 530 & 1372 & 1372 & 1320 & 1320 \\
\hline
\end{tabular}

$\theta=$ Ángulo en el plano longitudinal-tangencial; $v_{u s}=$ Velocidad del ultrasonido; $E_{u s}=$ Módulo de elasticidad;

$\mathrm{L}=$ Dirección longitudinal; $\mathrm{T}=$ Dirección tangencial.

La relación de anisotropía entre las direcciones longitudinal y tangencial, fueron para la velocidad del ultrasonido de $T$. plicata de 4,68 y para la de A. saccharum de 4,21. Estos valores concuerdan con lo reportado por Sotomayor et al. (2010) para maderas gimnospermas, usando la misma técnica de ultrasonido empleada en esta investigación. Asimismo, estos autores reportan relaciones de anisotropía para los módulos de elasticidad con ultrasonido de 20,73 magnitud comparable a los de esta investigación determinadas para T. plicata de 20,99 y para A. saccharum de 18,7.

Bucur y Declercq (2006) reportan para Picea abies una relación de anisotropía entre las direcciones longitudinal y tangencial de 4,65. De acuerdo con Bucur y Declercq (2006), valores altos de esta propiedad se utilizan como un criterio de selección para maderas con vocación para la fabricación 
de instrumentos musicales. De la misma manera, Hasegawa et al. (2011) reportan coeficientes de anisotropía para Cryptomeria japonica de 3,12 y para Chamaecyparis obtusa de 3,23 para mediciones en la madera con ultrasonido.

Efectivamente, las maderas de gimnospermas presentan relaciones de anisotropía mayores a las de especies angiospermas. Brémaud et al. (2011), reportan para el módulo de elasticidad determinado con métodos dinámicos, relaciones de anisotropía para las direcciones longitudinal-tangencial de 18,5 para maderas de gimnospermas y de 15,4 para maderas de angiospermas.

La extensa biodiversidad natural en las plantas gimnospermas y angiospermas, resulta en una amplia variación en las características tecnológicas propias a cada especie de madera. De tal suerte, que la construcción de teorías derivadas de investigación en ciencias de la madera, se rige por el principio de la caracterización de caso por caso, especie por especie, para finalmente proponer el agrupamiento de especies con una tendencia probabilística similar en su comportamiento y/o respuesta mecánicos. Este argumento está documentado para la clasificación de madera con vocación para la fabricación de instrumentos musicales por Yoshikawa (2007) y Brémaud (2012a).

De aquí, que para la especificación del módulo de elasticidad de una especie, es necesario asociar al valor de la característica, algunos parámetros de referencia. Esta información complementaria es, entre otras, la densidad del material estudiado, su contenido de humedad al momento del ensayo, la dirección de la solicitación, el tipo de prueba realizada y el tamaño de la muestra observada. De no ser así, es difícil comparar resultados entre diferentes especies y protocolos experimentales. Por ejemplo, Se Golpayegani et al. (2012) determinan en madera de Morus alba, con densidad de $556 \mathrm{~kg} / \mathrm{m}^{3}$ y un contenido de humedad de $7 \%$, un módulo de elasticidad longitudinal de 7066 MPa y para la dirección tangencial un módulo de $883 \mathrm{MPa}$, resultando en una relación de anisotropía T/L de 8, magnitud menor que las aquí reportadas, aun ponderándola con la densidad y el contenido de humedad. No obstante, esta madera es utilizada tradicionalmente para fabricar instrumentos musicales (Bucur 2006). Siguiendo siempre a Se Golpayegani et al. (2012), la madera con mejores aptitudes musicales debe presentar importantes relaciones de anisotropía, de este modo, las altas relaciones de anisotropía de la madera de T. plicata y de A. saccharum, confirman su vocación de empleo en instrumentos musicales.

Las tendencias de la velocidad del ultrasonido y del módulo de elasticidad en función del ángulo de rotación en el plano longitudinal-tangencial, se presentan en las Figuras 2 y 3. Las curvas de aproximación calculadas con el modelo de anisotropía (Fórmula 2), para T. plicata, derivan de una constante $\mathrm{n}=2,133$ para $v_{u s}$ con un $\mathrm{R}^{2}{ }_{\text {ajustado }}=0,978 \mathrm{y} \mathrm{n}=2,645$ para $E_{u s}$ con un $\mathrm{R}^{2}$ ajustado $=0,978$. En la Figura 3, se presentan las tendencias para $A$. saccharum. Las constantes $\mathrm{n}$ son: para $\mathrm{v}_{\text {us }}$ de 1,665 con un $\mathrm{R}^{2}{ }_{\text {ajustado }}=0,999$ y para $E_{u s}$ de 2,111 con $\mathrm{R}^{2}$ ajustado $=0,997$.

De tal forma, que el modelo de anisotropía de la madera se puede definir, para la madera de $T$. plicata como:

$$
\begin{aligned}
E_{u s \theta} & =\frac{E_{u s L} E_{u s T}}{E_{u s L} \operatorname{sen}^{2,645} \theta+E_{u s T} \cos ^{2,645} \theta} \\
V_{u s \theta} & =\frac{V_{u s L} V_{u s T}}{V_{u s L} \operatorname{sen}^{2,133} \theta+V_{u s T} \cos ^{2,133} \theta} \\
V_{u s \theta} & =\frac{V_{u s L} V_{u s T}}{V_{u s L} \operatorname{sen}^{1,665} \theta+V_{u s T} \cos ^{1,665} \theta}
\end{aligned}
$$

Y para la madera de $A$. saccharum, el modelo de anisotropía de la madera se puede definir como:

$$
E_{u s \theta}=\frac{E_{u s L} E_{u s T}}{E_{u s L} \operatorname{sen}^{2,111} \theta+E_{u s T} \cos ^{2,111} \theta}
$$

En las ecuaciones 3, 4, 5 y $6: \mathrm{v}_{\text {us }}=$ Velocidad del ultrasonido $(\mathrm{m} / \mathrm{s}) ; E_{u s}=$ Módulo de elasticidad 
(MPa): $\mathrm{L}=$ Dirección longitudinal; $\mathrm{T}=$ Dirección tangencial; y $\theta=$ Ángulo entre las direcciones longitudinal y tangencial $\left(^{\circ}\right)$.

Las predicciones teóricas de los modelos explicados por las fórmulas (3), (4), (5) y (6), fueron graficadas en las Figuras 2 y 3. En las mismas Figuras, cada punto correspondiente a los valores experimentales, representa el promedio de las mediciones en 32 probetas.
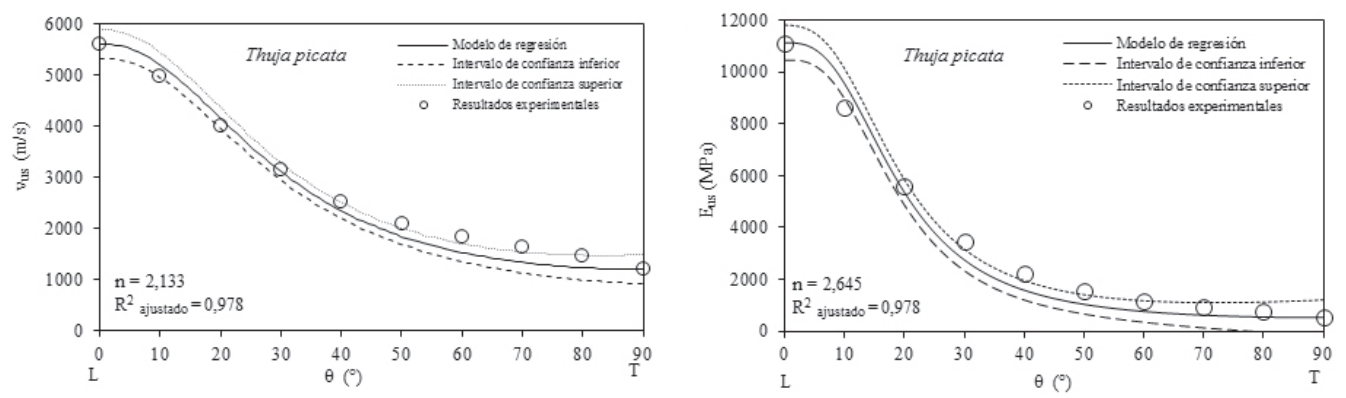

Figura 2. Tendencias de los resultados experimentales y previsiones del modelo de anisotropía para la velocidad del ultrasonido $\left(v_{u s}\right)$ y para el módulo de elasticidad $\left(E_{u s}\right)$ de $T$. plicata, en función del ángulo $(\theta) . \mathrm{L}=$ Dirección longitudinal, $\mathrm{T}=$ Dirección tangencial.
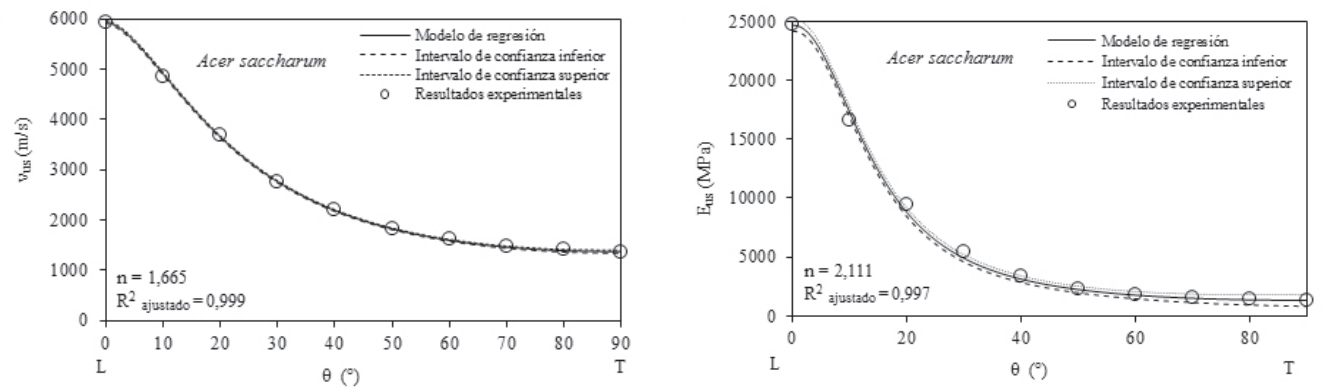

Figura 3. Tendencias de los resultados experimentales y previsiones del modelo de anisotropía para la velocidad del ultrasonido $\left(v_{u s}\right)$ y el módulo de elasticidad $\left(E_{u s}\right)$ de $A$. saccharum, en función del ángulo $(\theta) . \mathrm{L}=$ Dirección longitudinal, $\mathrm{T}=$ Dirección tangencial.

Las tendencias para A. saccharum, se aproximan con los datos experimentales. En contraste, las de T. plicata, se separan de las predicciones teóricas. Durante las pruebas de ultrasonido, se observó que las mediciones en la madera de T. plicata variaban más, comparativamente con las de A. saccharum. Esta particularidad es mostrada en la Figura 4, donde se presentan los coeficientes de variación de la velocidad del ultrasonido en función de la rotación en el plano Longitudinal-Tangencial. En efecto, mientras que el coeficiente de variación de $A$. saccharum se mantiene relativamente constante, para $T$. plicata varía y tiende a aumentar cuando se aproximan las mediciones en la dirección radial. Gonçalves et al. (2011 y 2014) aplicando ultrasonido a once maderas y utilizando probetas de forma circular, semejantes a las de la presente investigación, obtienen coeficientes de variación para la velocidad del ultrasonido en la dirección longitudinal entre 1,2 \% y 3,6 \% y para la dirección tangencial entre $3 \%$ y $3,8 \%$ variaciones menores a las aquí obtenidas. 
Kránitz et al. (2014) reportan para Picea abies, coeficientes de variación de 10,1\% para un módulo de elasticidad correspondiente a la dirección longitudinal, de $24090 \mathrm{MPa}$ y para la dirección tangencial, un coeficiente de variación de $20,2 \%$ pertinente a un módulo de elasticidad de $786 \mathrm{MPa}$. Estos resultados son compatibles con los desplegados en la Figura 4, para los coeficientes de variación y con los mostrados en la Tabla 1 para los módulos de elasticidad y relativamente comparables con los de la madera de $P$. abies, madera que es usualmente empleada como referencia en investigación del comportamiento acústico de la madera (Brémaud 2012b).

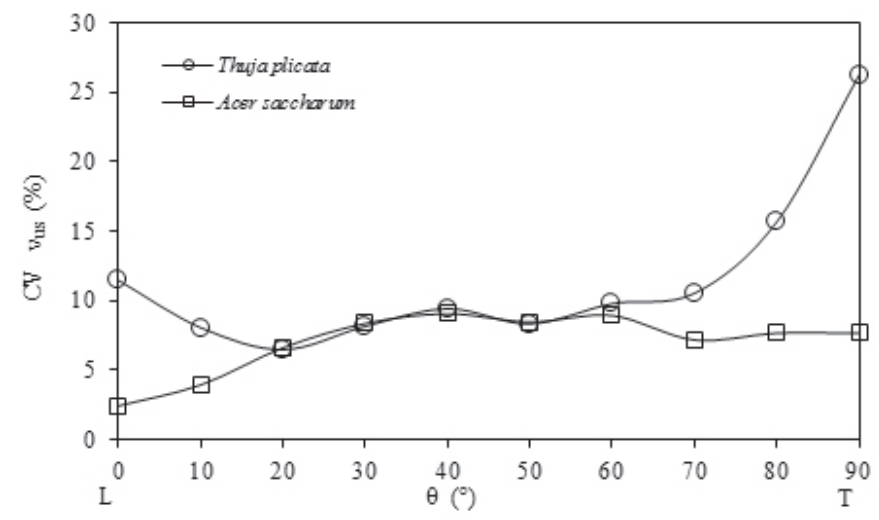

Figura 4. Coeficiente de variación $(\mathrm{CV})$ de la velocidad del ultrasonido $\left(\mathrm{v}_{\mathrm{us}}\right)$ en función del ángulo $(\theta)$ en el plano Longitudinal (L) - Tangencial (T).

\section{CONCLUSIONES}

Utilizando ultrasonido, se calcularon los módulos de elasticidad de las maderas de T. plicata y A. saccharum para 10 direcciones en el plano longitudinal-tangencial. Los resultados mostraron una variación gradual en los valores del módulo de elasticidad al pasar de la dirección longitudinal a la tangencial, de esta manera se confirmó experimentalmente el comportamiento anisotrópico de las maderas estudiadas.

Los resultados demostraron que no existen diferencias estadísticamente significativas entre los valores experimentales y los valores calculados con el modelo teórico de anisotropía para el módulo de elasticidad.

Dado que la densidad de la madera se consideró constante, la ecuación utilizada para calcular el módulo de elasticidad dependió exclusivamente de la velocidad del ultrasonido. De tal forma que se concluye que la velocidad del ultrasonido y el módulo de elasticidad siguen un patrón anisotrópico que puede ser inferido con un modelo matemático y con un nivel razonable de precisión, verificando de esta manera la hipótesis de la investigación. 


\section{REFERENCIAS}

Armstrong, J.P.; Patterson, D.W.; Sneckenberger, J.E. 1991. Comparison of three equations for predicting stress wave velocity as a function of grain angle. Wood and Fiber Science 23(1):32-43.

Brémaud, I. 2012a. Acoustical properties of wood in string instruments soundboards and tuned idiophones: Biological and cultural diversity. Journal of the Acoustical Society of America 131(1):807818.

Brémaud, I. 2012b. What do we know on "resonance wood" properties? Selective review and ongoing research. Societe Francaise d'Acoustique. Proceedings of the Acoustics 2012. Nantes Conference: $2759-2764$.

Brémaud, I.; El Kaïm, Y.; Guibal, D.; Minato, M.; Thibaut, B.; Gril, J. 2012. Characterization and categorization of the diversity in viscoelastic vibrational properties between 98 wood types. Annals of Forest Science 69(3):373-386.

Brémaud, I.; Gril, J.; Thibaut, B. 2011. Anisotropy of wood vibrational properties: dependence on grain angle and review of literature data. Wood Science and Technology 45(4):735-754.

Bucur, V. 2006. Acoustics of wood. Springer-Verlag, Berlin.

Bucur, V.; Declercq, N. 2006. The anisotropy of biological composites studied with ultrasonic technique. Ultrasonics 44:829-831.

De Oliveira, F.G.R.; Candian, M.; Lucchette, F.F.; Salgon, J.L.; Sales, A. 2005. A technical note on the relationship between ultrasonic velocity and moisture content of Brazilian hardwood (Goupia glabra). Building and Environment 40(2):297-300.

Forest Products Laboratory. 2010. Wood handbook-Wood as an engineering material. General Technical Report FPL-GTR-190. U.S. Department of Agriculture, Forest Service. Forest Products Laboratory. Madison.

Gonçalves, R.; Trinca, A.J.; Dos Santos Ferreira, G.C. 2011. Effect of coupling media on velocity and attenuation of ultrasonic waves in Brazilian wood. Journal of Wood Science 57(4):282287.

Gonçalves, R.; Trinca, A.J.; Pellis, B.P. 2014. Elastic constants of wood determined by ultrasound using three geometries of specimens. Wood Science and Technology 48(2):269-287.

Hasegawa, M.; Takata, M.; Matsumura, J.; Oda, K. 2011. Effect of wood properties on withintree variation in ultrasonic wave velocity in softwood. Ultrasonics 51(3):296-302.

International Organization for Standardization. ISO. 2014a. Physical and mechanical properties of wood - Test methods for small clear wood specimens - Part 1: Determination of moisture content for physical and mechanical tests. ISO 13061-1:2014. ISO Catalog 79 Wood technology; 79.040 Wood, sawlogs and saw timber. Brussels.

International Organization for Standardization. ISO. 2014b. Physical and mechanical properties of wood - Test methods for small clear wood specimens - Part 2: Determination of density for physical and mechanical tests. ISO 13061-2:2014. ISO Catalog 79 Wood technology; 79.040 Wood, sawlogs and saw timber. Brussels.

Kabir, M.F. 2001. Prediction of Ultrasonic Properties from Grain Angle. Journal of the Institute of Wood Science 15(5):235-246. 
Keunecke, D.; Sonderegger, W.; Pereteanu, K.; Lüthi, T.; Niemz, P. 2007. Determination of young's and shear moduli of common yew and Norway spruce by means of ultrasonic waves. Wood Science and Technology 41(4):309-327.

Kránitz, K.; Deublein, M.; Niemz, P. 2014. Determination of dynamic elastic moduli and shear moduli of aged wood by means of ultrasonic devices. Materials and Structures 47(6):925-936.

Mackerle, J. 2005. Finite element analyses in wood research: a bibliography. Wood Science and Technology 39(7):579-600.

Nadir, Y.; Nagarajan, P.; Midhun, A.J. 2014. Measuring elastic constants of Hevea brasiliensis using compression and Iosipescu shear test. European Journal of Wood and Wood Products 72(6):749758 .

Ozyhar, T.; Hering, S.; Sanabria, S.J.; Niemz, P. 2013. Determining moisture-dependent elastic characteristics of beech wood by means of ultrasonic waves. Wood Science and Technology 47(2):329341 .

Pellerin, R.F.; Ross, R.J. 2002. Nondestructive Evaluation of Wood. Forest Products Society. Peachtree Corners. 210 p.

Se Golpayegani, A.; Brémaud, I.; Gril, J.; Thevenon, M-F.; Arnould, O.; Pourtahmasi, K. 2012. Effect of extractions on dynamic mechanical properties of white mulberry (Morus alba). Journal of Wood Science 58(2):153-162.

Sonderegger, W.; Martienssen, A.; Nitsche, C.; Ozyhar, T.; Kaliske, M.; Niemz, P. 2013. Investigations on the physical and mechanical behavior of sycamore maple (Acer pseudoplatanus L.). European Journal of Wood and Wood Products 71(1):91-99.

Sotomayor Castellanos, J.R.; Guridi Gomez, L.I.; Garcia Moreno, T. 2010. Características acústicas de la madera de 152 especies mexicanas. Velocidad del ultrasonido, módulo de elasticidad, índice material y factor de calidad. Base de datos. Investigación e Ingeniería de la Madera 6(1):3-32.

Sotomayor Castellanos, J.R.; Ramírez Pérez, M. 2013. Densidad y características higroscópicas de maderas mexicanas. Base de datos y criterios de clasificación. Investigación e Ingeniería de la Madera 9(3):3-29.

Sotomayor Castellanos, J.R.; Ramírez Pérez, M. 2014. Características físicas de 12 maderas mexicanas. Investigación e Ingeniería de la Madera 10(1):4-35.

Tankut, N.; Tankut, A.N.; Zor, M. 2014. Finite Element Analysis of Wood Materials. Drvna Industrija 65(2):159-171.

Vázquez, C.; Gonçalves, R.; Bertoldo, C.; Baño, V.; Vega, A.; Crespo, J.; Guaita, M. 2015. Determination of the mechanical properties of Castanea sativa Mill. using ultrasonic wave propagation and comparison with static compression and bending methods. Wood Science and Technology 49(3):607-622.

Wegst, U.G.K. 2006. Wood for sound. American Journal of Botany 93(10):1439-1448.

Xu, H.; Xu, G.; Wang, L.; Yu, L. 2014. Propagation behavior of acoustic wave in wood. Journal of Forestry Research 25(3):671-676.

Yanagida, H.; Tamura, Y.; Kim, K.M.; Lee, J.J. 2007. Development of Ultrasonic Time-ofFlight Computed Tomography for Hard Wood with Anisotropic Acoustic Property. Japanese Journal of Applied Physics 46(8A):5321-5325.

Yoshikawa, S. 2007. Acoustical classification of woods for string instruments. Journal of the Acoustical Society of America 122(1):568-573. 